\title{
Epidemiology and Genetic Diversity of Colistin Nonsusceptible Nosocomial Acinetobacter baumannii Strains from Russia for 2013-2014
}

\author{
Eugene A. Sheck, Mikhail V. Edelstein, Marina V. Sukhorukova, Natali V. Ivanchik, \\ Elena Yu. Skleenova, Andrey V. Dekhnich, Ilya S. Azizov, and Roman S. Kozlov \\ Institute of Antimicrobial Chemotherapy (IAC), Smolensk State Medical University (SSMU), Kirova St. 46a, \\ Smolensk 214019, Russia \\ Correspondence should be addressed to Eugene A. Sheck; evgeniy.sheck@antibiotic.ru
}

Received 29 June 2017; Accepted 13 September 2017; Published 17 October 2017

Academic Editor: Branka Bedenić

Copyright (C) 2017 Eugene A. Sheck et al. This is an open access article distributed under the Creative Commons Attribution License, which permits unrestricted use, distribution, and reproduction in any medium, provided the original work is properly cited.

\begin{abstract}
A high level of resistance to carbapenems in Acinetobacter baumannii strains severely limits therapeutic possibilities. Colistin is the last resort drug against such strains, although the cases of resistance to this drug have become more frequent. This article presents the epidemiological features and genetic diversity of colistin nonsusceptible A. baumannii strains collected as part of a national multicenter epidemiological study of the antibiotic resistance of pathogens of nosocomial infections (MARATHON), which was conducted in 2013-2014 in Russia. A total of 527 A. baumannii isolates were collected, 10 (1.9\%) of which were nonsusceptible to colistin. The majority of nonsusceptible A. baumannii isolates to colistin showed resistance to carbapenems and had the genes of the acquired OXA-40-like carbapenemases $(n=6)$. In one case, a combination of OXA-23-like + OXA-40-like $(n=1)$ genes was identified. One strain had the multidrug-resistant (MDR) phenotype, 6 isolates had extensively drug-resistant (XDR) phenotype, and 3 isolates had pandrug-resistant (PDR) phenotype. Among the colistin nonsusceptible A. baumannii isolates, 6 individual genotypes were identified, most of which belonged to successful international clones $\left(\mathrm{CC} 92^{\mathrm{OXF}} / \mathrm{CC} 2^{\mathrm{PAS}}, n=4 ; \mathrm{CC} 944^{\mathrm{OXF}} / \mathrm{ST} 78^{\mathrm{PAS}}\right.$, $n=4$; $\left.\mathrm{CC} 109^{\mathrm{OXF}} / \mathrm{CC}^{\mathrm{PAS}}, n=1\right)$.
\end{abstract}

\section{Introduction}

Acinetobacter baumannii is one of the most troublesome pathogens of nosocomial infections $[1,2]$. This pathogen is characterized by intrinsic resistance to a number of drugs, as well as an outstanding ability to acquire the determinants of antibiotic resistance through horizontal gene transfer, which led to a high level of $A$. baumannii strains resistance to almost all available antibiotics [3, 4]. A high level of in vitro sensitivity is retained only for colistin [5], which makes it possible to use it against the extensively drug-resistant A. baumannii strains [6]. At the same time, the cases of resistance to colistin become more frequent [7-9], which determines the necessity to monitor resistance to colistin among nosocomial $A$. baumannii strains. In addition, the spread of the multidrug-resistant (MDR) phenotype is mainly related to the spread of international high-risk clones and the horizontal transfer of antibiotic resistance genes [10, 11], which provoke the interest in studying the molecular epidemiology of colistin nonsusceptible strains. The purpose of the research is to study the epidemiology and genetic diversity of colistin nonsusceptible A. baumannii strains, isolated from clinical samples of hospitalized patients in different regions of Russia in 2013-14.

\section{Materials and Methods}

Clinical strains of microorganisms were collected as part of a multicenter epidemiological surveillance study of the antibiotic resistance of nosocomial pathogens (MARATHON) [12] in 21 cities of Russia from January 2013 to December 2014. 527 nosocomial isolates of $A$. baumannii were collected. In this study, only colistin nonsusceptible strains (MIC $>2 \mathrm{mg} / \mathrm{L})$ were analyzed. 
Species identification was performed by the matrixassisted laser desorption/ionization time-of-flight mass spectrometry (MALDI-ToF MS) using Microflex LT mass spectrometer and MALDI Biotyper Compass software v. 4.1.70 (Bruker Daltonics, Germany). The value of "Score" $\geq 2.0$ was accepted as a measure for the reliable identification.

Minimal inhibitory concentrations of antimicrobials have been determined by broth microdilution method with Mueller Hinton broth (Oxoid, United Kingdom) in accordance with ISO 20776-1:2006 [13]. Interpretation of MIC in clinical susceptibility categories of $A$. baumannii isolates to antimicrobial agents was performed according to European Committee on Antimicrobial Susceptibility Testing (EUCAST) Breakpoint tables v.7.1 [14] (for aminoglycosides, carbapenems, ciprofloxacin, trimethoprim/sulfamethoxazole, and colistin) and also Clinical and Laboratory Standards Institute CLSI M100-S27 [15] (for penicillins with $\beta$-lactamase inhibitors and extended-spectrum cephalosporins). Isolates with tigecycline $\mathrm{MIC}$ of $>1 \mathrm{mg} / \mathrm{L}$ were considered insusceptible to this drug [16]. Escherichia coli ATCC ${ }^{\circledR} 25922$, E. coli ATCC $^{\circledR 35218}$, and Pseudomonas aeruginosa $\mathrm{ATCC}^{\circledR} 27853$ were used as control strains.

DNA extraction was performed by express method by using InstaGene ${ }^{\mathrm{TM}}$ matrix (Bio-Rad, USA). Samples of extracted DNA were stored at $-20^{\circ} \mathrm{C}$ before testing.

Detection of genes encoding the most common carbapenemases of class D (OXA-23, OXA-24/40, and OXA-58) and metal $\beta$-lactamases (VIM, IMP, and NDM) was carried out by real-time PCR using commercial kits “AmpliSens ${ }^{\circledR}$ MDR Acinetobacter-OXA-FL" and "AmpliSens ${ }^{\circledR}$ MDR MBL-FL" (InterLabService, Russia) and DTPrime 5X1 system (DNA Technology, Russia). A. baumannii, A. pittii, and $P$. aeruginosa strains from proper collection, producing known carbapenemases of the listed groups, were used as positive controls.

Genotyping was performed by single-nucleotide polymorphism (SNP) typing method [17]. Briefly, this method is based on analysis of 21 informative SNPs in 10 chromosomal loci (gltA, recA, cpn60, gyrB, gdhB, rpoD, fus A, pyrG, rplB, and $r p o B$ ) and is used at the University of Oxford and the Pasteur Institute multilocus sequence typing (MLST) schemes. $A$. baumannii strains of known sequence types from proper collection were used as positive controls. The comparison of received SNP profiles with MLST data was done using a web resource: http://snptab.antibiotic.ru [18]. Cluster analysis of SNP profiles was performed using PHYLOViZ 2.0 software (http://www.phyloviz.net/).

\section{Results and Discussion}

Ten of 527 (1.9\%) nosocomial colistin nonsusceptible $A$. baumannii isolates were isolated from 7 hospitals in 7 cities of Russia in 2013-2014. The results of antimicrobial susceptibility testing for these isolates are presented in Table 1. The MIC of colistin $4 \mathrm{mg} / \mathrm{L}$ was detected in 1 isolate, while the remaining isolates $(n=9)$ had high levels of resistance (MIC range: $32-256 \mathrm{mg} / \mathrm{L})$.

The majority of colistin nonsusceptible A. baumannii isolates were also resistant to carbapenems and were the

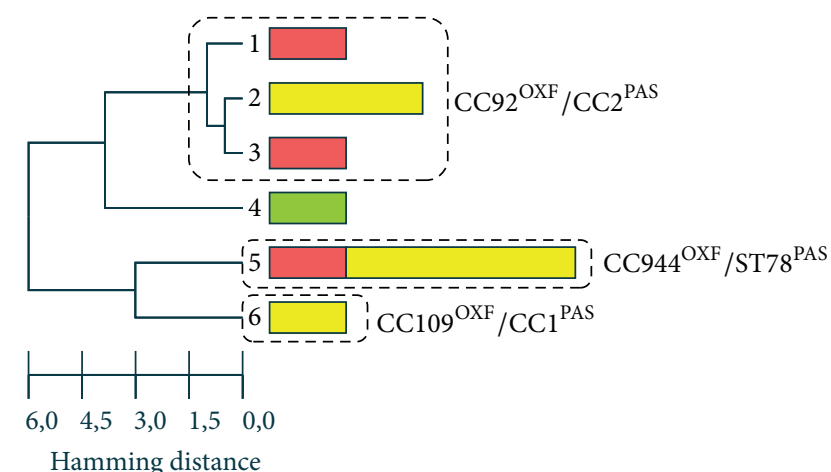

MDR

XDR

PDR

FIGURE 1: UPGMA clustering of SNP profiles of colistin nonsusceptible A. baumannii strains. The length of each rectangle depends on the number of isolates. International epidemic clones are marked with dashed lines. Color detailed the resistance categories (MDR, $\mathrm{XDR}$, and PDR) of isolates within each genotype.

carriers of genes of acquired OXA type carbapenemases, mainly OXA-40-like $(n=6)$. One isolate was shown to possess simultaneously genes encoding two different carbapenem hydrolyzing oxacillinases: OXA-23-like and OXA40-like. Expectedly high resistance was detected to all other $\beta$-lactams. At the same time, associated resistance to drugs of other groups with high MIC values is observed (range of gentamicin MIC: 32-256 mg/L; amikacin: $16-256 \mathrm{mg} / \mathrm{L}$; ciprofloxacin: $32-128 \mathrm{mg} / \mathrm{L})$. Only one tobramycin susceptible isolate was found (MIC $=1 \mathrm{mg} / \mathrm{L})$, while the MIC for the remaining isolates was in the range $16-256 \mathrm{mg} / \mathrm{L}$. Two isolates were susceptible to combination of trimethoprimsulfamethoxazole (MIC range: $1-2 \mathrm{mg} / \mathrm{L}$ ), while for the remaining isolates the MIC was in the range $4-128 \mathrm{mg} / \mathrm{L}$. Six isolates had tigecycline MICs of 2 to $8 \mathrm{mg} / \mathrm{L}$ and thus were considered as resistant.

In accordance with international criteria [19], 1 isolate was defined as multidrug-resistant (MDR), 6 isolates were defined as extensively drug-resistant (XDR), and 3 isolates were defined as pandrug-resistant (PDR).

Most infections associated with colistin nonsusceptible $A$. baumannii isolates were detected as single cases in different cities of Russia. An exception is Smolensk, from which 4 isolates were isolated in one hospital. In this connection, it seemed interesting to evaluate the population structure of studied strains.

Colistin nonsusceptible A. baumannii isolates belonged to 6 genotypes (Figure 1). Three genotypes differed from each other in no more than 2 positions and were united in a single genetic cluster as related genotypes. This cluster included 4 isolates from 4 cities and corresponded to the international clone $\mathrm{CC} 92^{\mathrm{OXF}} / \mathrm{CC} 2^{\mathrm{PAS}}$ (according to the MLST schemes of the University of Oxford and the Pasteur Institute, resp.). Another genotype included 4 isolates from 3 cities and corresponded to $\mathrm{CC} 944^{\mathrm{OXF}} / \mathrm{ST} 78^{\mathrm{PAS}}$. The strains of this 


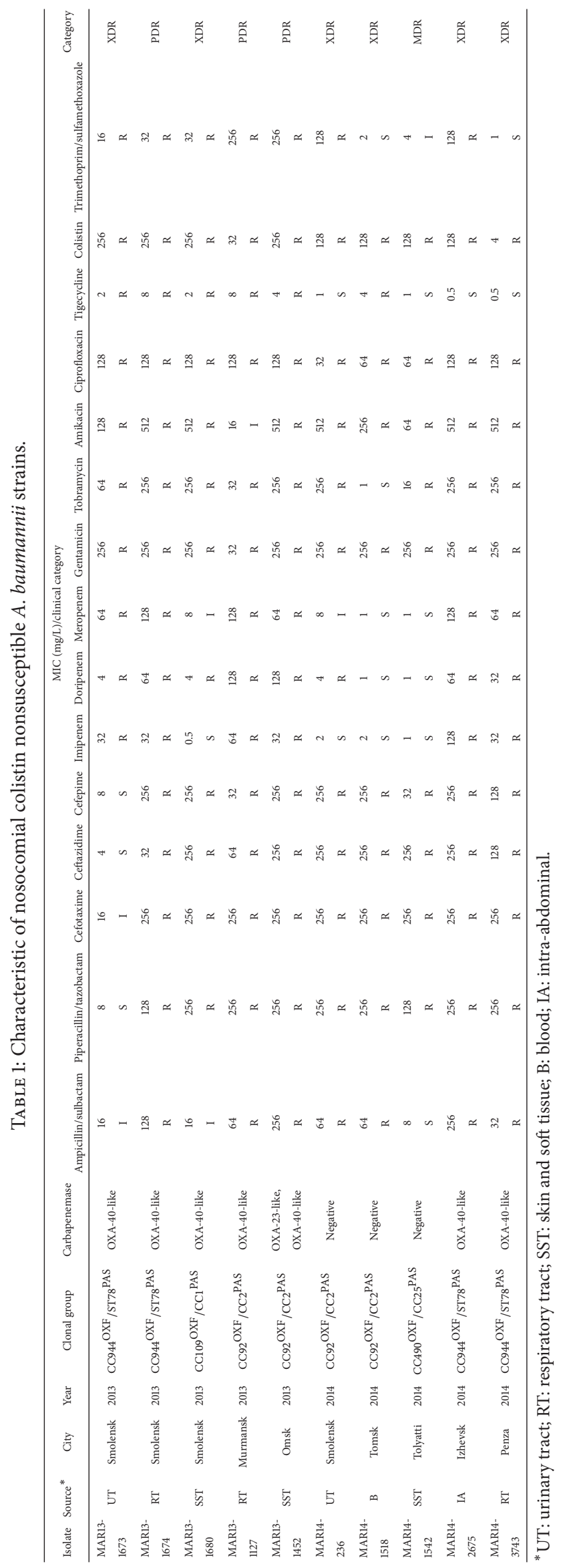


genetic lineage are widespread in the territory of Russia and Belarus [12] and, according to the literature, were found in Italy, the US, and Germany [20-23]. This allows considering this genotype as a new international clone. Two isolates belonged to individual genotypes: one of them corresponded to the international clone $\mathrm{CC} 109^{\mathrm{OXF}} / \mathrm{CC}^{\mathrm{PAS}}$ and the other to the clonal complex CC490 ${ }^{\mathrm{OXF}} / \mathrm{CC} 25^{\mathrm{PAS}}$. All isolates with $\mathrm{XDR}$ and $\mathrm{PDR}$ phenotypes belonged to the international epidemic clones CC92 ${ }^{\mathrm{OXF}} / \mathrm{CC} 2^{\mathrm{PAS}}$, CC944 ${ }^{\mathrm{OXF}} / \mathrm{ST} 78^{\mathrm{PAS}}$, and $\mathrm{CC} 109^{\mathrm{OXF}} / \mathrm{CC} 1^{\mathrm{PAS}}$.

\section{Conclusion}

Thus, colistin nonsusceptible A. baumannii strains described in this study relate to different genetic lineages and mainly belong to distinct international high-risk clones. The accumulation of molecular typing data is an important element in understanding the roots and epidemiology of colistin resistance and in predicting its further spread.

\section{Conflicts of Interest}

The authors declare that they have no conflicts of interest.

\section{References}

[1] L. Dijkshoorn, A. Nemec, and H. Seifert, "An increasing threat in hospitals: multidrug-resistant Acinetobacter baumannii," Nature Reviews Microbiology, vol. 5, no. 12, pp. 939-951, 2007.

[2] M. Kempf and J.-M. Rolain, "Emergence of resistance to carbapenems in Acinetobacter baumannii in Europe: Clinical impact and therapeutic options," International Journal of Antimicrobial Agents, vol. 39, no. 2, pp. 105-114, 2012.

[3] R. A. Bonnin, P. Nordmann, and L. Poirel, "Screening and deciphering antibiotic resistance in Acinetobacter baumannii: A state of the art," Expert Review of Anti-infective Therapy, vol. 11, no. 6, pp. 571-583, 2013.

[4] L. C. S. Antunes, P. Visca, and K. J. Towner, "Acinetobacter baumannii: evolution of a global pathogen," Pathogens and Disease, vol. 71, no. 3, pp. 292-301, 2014.

[5] European Centre for Disease Prevention and Control, "Antimicrobial resistance surveillance in Europe 2015," Annual Report of the European Antimicrobial Resistance Surveillance Network (EARS-Net), European Centre for Disease ECDC, Stockholm, Sweden, 2017.

[6] I. Karaiskos and H. Giamarellou, "Multidrug-resistant and extensively drug-resistant Gram-negative pathogens: current and emerging therapeutic approaches," Expert Opinion on Pharmacotherapy, vol. 15, no. 10, pp. 1351-1370, 2014.

[7] I. Karaiskos, M. Souli, I. Galani, and H. Giamarellou, "Colistin: still a lifesaver for the 21st century?" Expert Opinion on Drug Metabolism \& Toxicology, vol. 13, no. 1, pp. 59-71, 2017.

[8] K. Jeannot, A. Bolard, and P. Plésiat, "Resistance to polymyxins in Gram-negative organisms," International Journal of Antimicrobial Agents, vol. 49, no. 5, pp. 526-535, 2017.

[9] Y. Cai, D. Chai, R. Wang, B. Liang, and N. Bai, "Colistin resistance of Acinetobacter baumannii: clinical reports, mechanisms and antimicrobial strategies," Journal of Antimicrobial Chemotherapy, vol. 67, no. 7, pp. 1607-1615, 2012.
[10] N. Karah, A. Sundsfjord, K. Towner, and $\varnothing$. Samuelsen, "Insights into the global molecular epidemiology of carbapenem non-susceptible clones of Acinetobacter baumannii," Drug Resistance Updates, vol. 15, no. 4, pp. 237-247, 2012.

[11] R. Zarrilli, S. Pournaras, M. Giannouli, and A. Tsakris, "Global evolution of multidrug-resistant Acinetobacter baumannii clonal lineages," International Journal of Antimicrobial Agents, vol. 41, no. 1, pp. 11-19, 2013.

[12] M. V. Sukhorukova, M. V. Edelstein, E. Yu. Skleenova et al., "Antimicrobial resistance of nosocomial Acinetobacter spp. isolates in Russia: results of multicenter epidemiological study ńMARATHONż 2013-2014," Clinical Microbiology and Antimicrobial Chemotherapy, vol. 19, no. 1, pp. 42-48, 2017.

[13] ISO 20776-1:2006, "Clinical laboratory testing and in vitro diagnostic test systems - Susceptibility testing of infectious agents and evaluation of performance of antimicrobial susceptibility test devices - Part 1 : Reference method for testing the in vitro activity of antimicrobial agents against rapidly growing aerobic bacteria involved in infectious diseases".

[14] European Committee on Antimicrobial Susceptibility testing (EUCAST), "Breakpoint tables for interpretation of MICs and zone diameters," Ver. 7.1, 2017, http://www.eucast.org/clinical_ breakpoints/.

[15] Clinical and Laboratory Standards Institute, Performance Standards for Antimicrobial Susceptibility Testing; Seventeenth Information Supplement, CLSI document M100-S17, Clinical and Laboratory Standards Institute, Wayne, Pa, USA, 2011, http://clsi.org/m100/.

[16] S. Pournaras, V. Koumaki, V. Gennimata, E. Kouskouni, and A. Tsakris, "In vitro activity of tigecycline against Acinetobacter baumannii: Global epidemiology and resistance mechanisms," Advances in Experimental Medicine and Biology, vol. 897, pp. 114, 2016.

[17] E. Sheck, A. Fedintsev, E. Skleenova, A. Martinovich, and M. Edelstein, "Development of a high-throughput single nucleotide polymorphism typing method for Acinetobacter baumannii (SNPTAb," in Proceedings of the P0079, 25th European Congress of Clinical Microbiology and Infectious Diseases (ECCMID, pp. 25-28, 2015.

[18] A. Fedintsev, E. Sheck, A. Avramenko, I. Trushin, and M. Edelstein, "Development of an online database and web application for analysis of SNP typing data of Acinetobacter baumannii EP0313," in Proceedings of the 26th European Congress of Clinical Microbiology and Infectious Diseases (ECCMID), vol. 38, ESCMID, Amsterdam, Netherlands, April 2016.

[19] A.-P. Magiorakos, A. Srinivasan, R. B. Carey et al., "Multidrugresistant, extensively drug-resistant and pandrug-resistant bacteria: an international expert proposal for interim standard definitions for acquired resistance," Clinical Microbiology and Infection, vol. 18, no. 3, pp. 268-281, 2012.

[20] M. Giannouli, S. Cuccurullo, V. Crivaro et al., "Molecular epidemiology of multidrug-resistant acinetobacter baumannii in a tertiary care hospital in Naples, Italy, shows the emergence of a novel epidemic clone," Journal of Clinical Microbiology, vol. 48, no. 4, pp. 1223-1230, 2010.

[21] M. Giannouli, L. C. S. Antunes, V. Marchetti, M. Triassi, P. Visca, and R. Zarrilli, "Virulence-related traits of epidemic Acinetobacter baumannii strains belonging to the international clonal lineages I-III and to the emerging genotypes ST25 and ST78," BMC Infectious Diseases, vol. 13, no. 1, article no. 282, 2013. 
[22] L. S. Munoz-Price, K. Arheart, P. Nordmann et al., "Eighteen years of experience with acinetobacter baumannii in a tertiary care hospital ${ }^{\star}$," Critical Care Medicine, vol. 41, no. 12, pp. 27332742, 2013.

[23] Y. Pfeifer, K.-P. Hunfeld, S. Borgmann et al., "Carbapenemresistant Acinetobacter baumannii ST78 with OXA-72 carbapenemase and ESBL gene blaCTX-M-115," Journal of Antimicrobial Chemotherapy, vol. 71, no. 5, Article ID dkv462, pp. 14261432, 2016. 


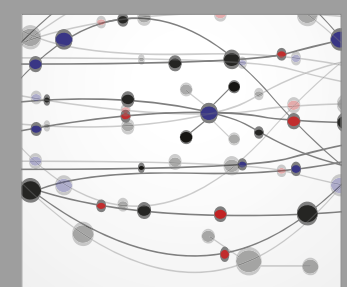

The Scientific World Journal
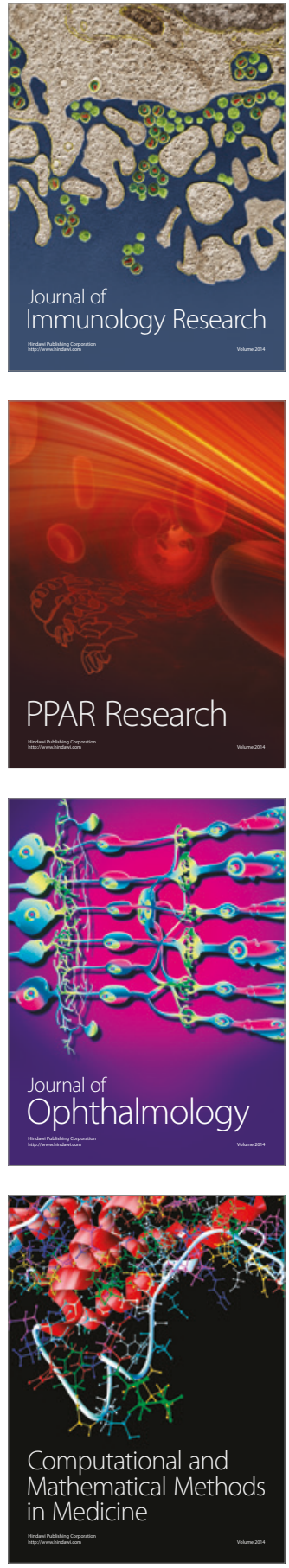

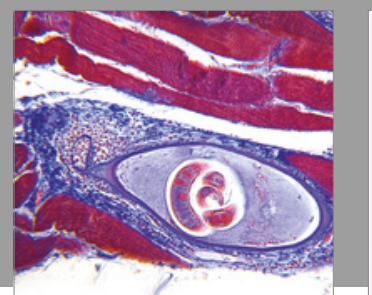

Gastroenterology Research and Practice
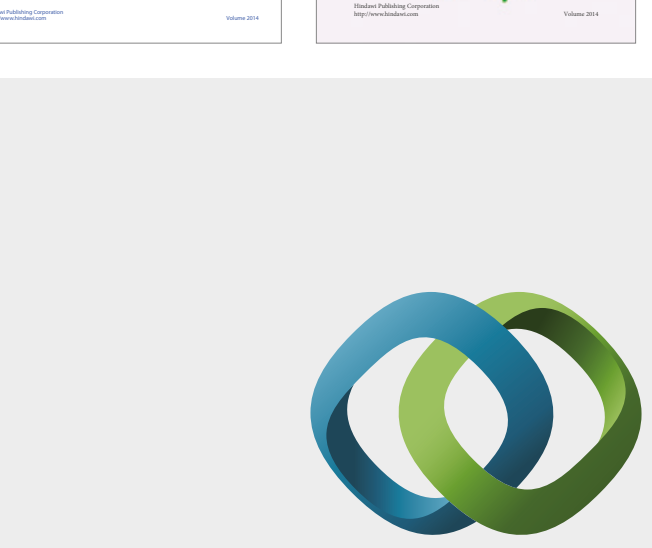

\section{Hindawi}

Submit your manuscripts at

https://www.hindawi.com
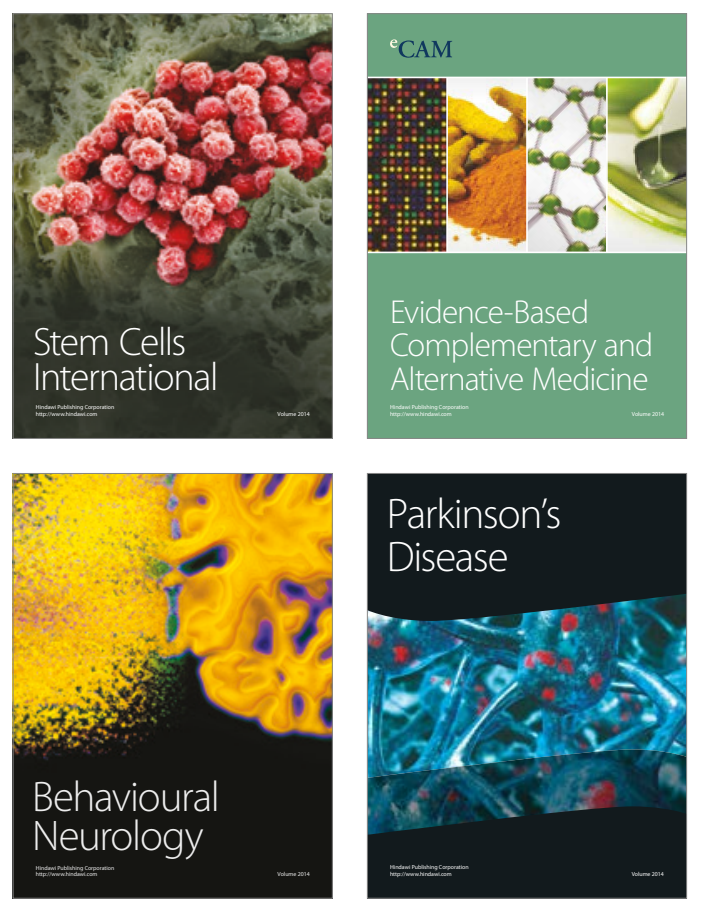
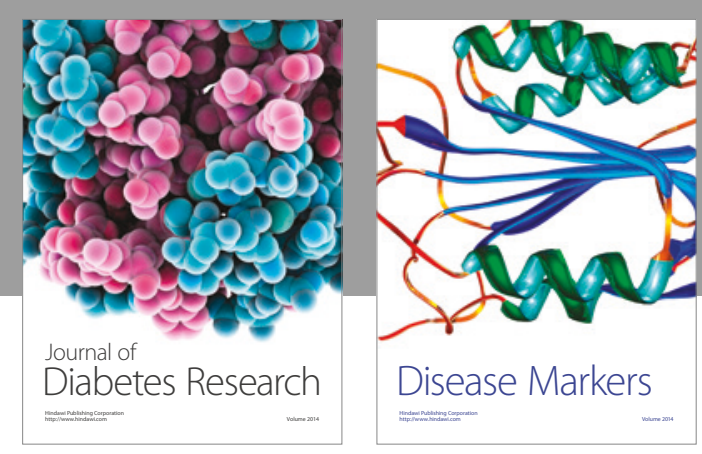

Disease Markers
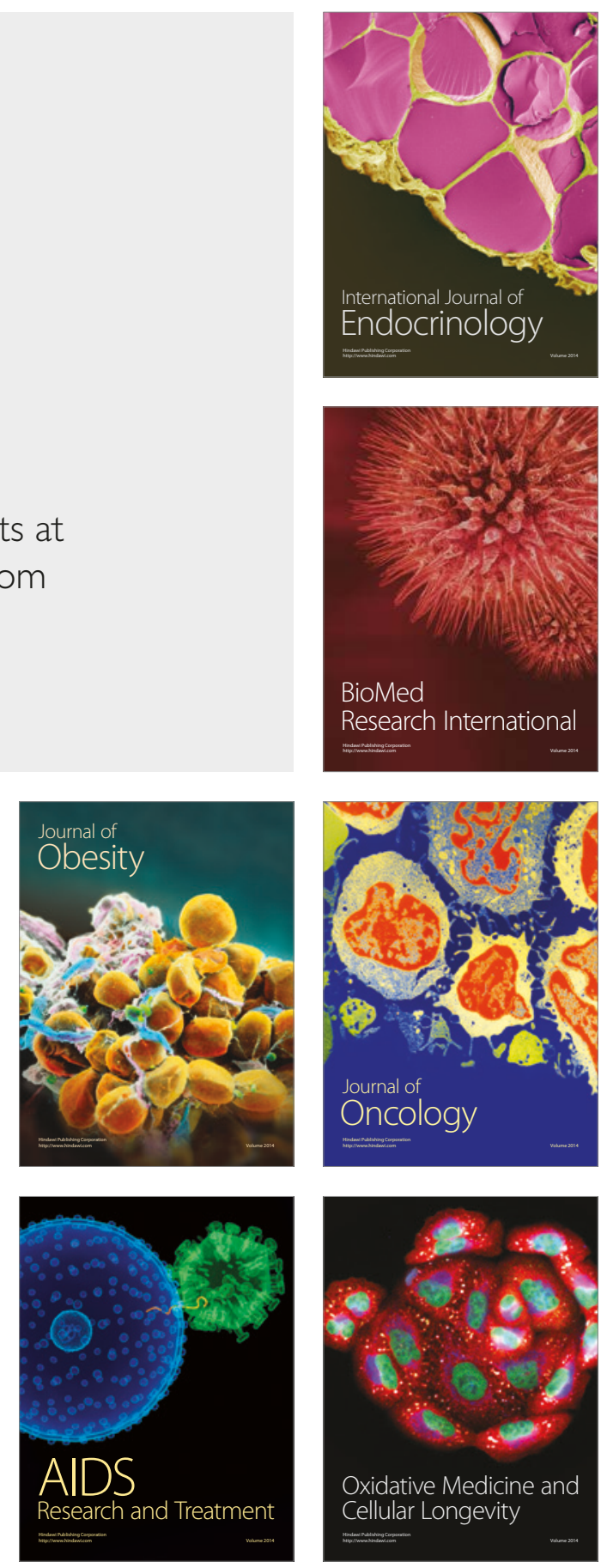